
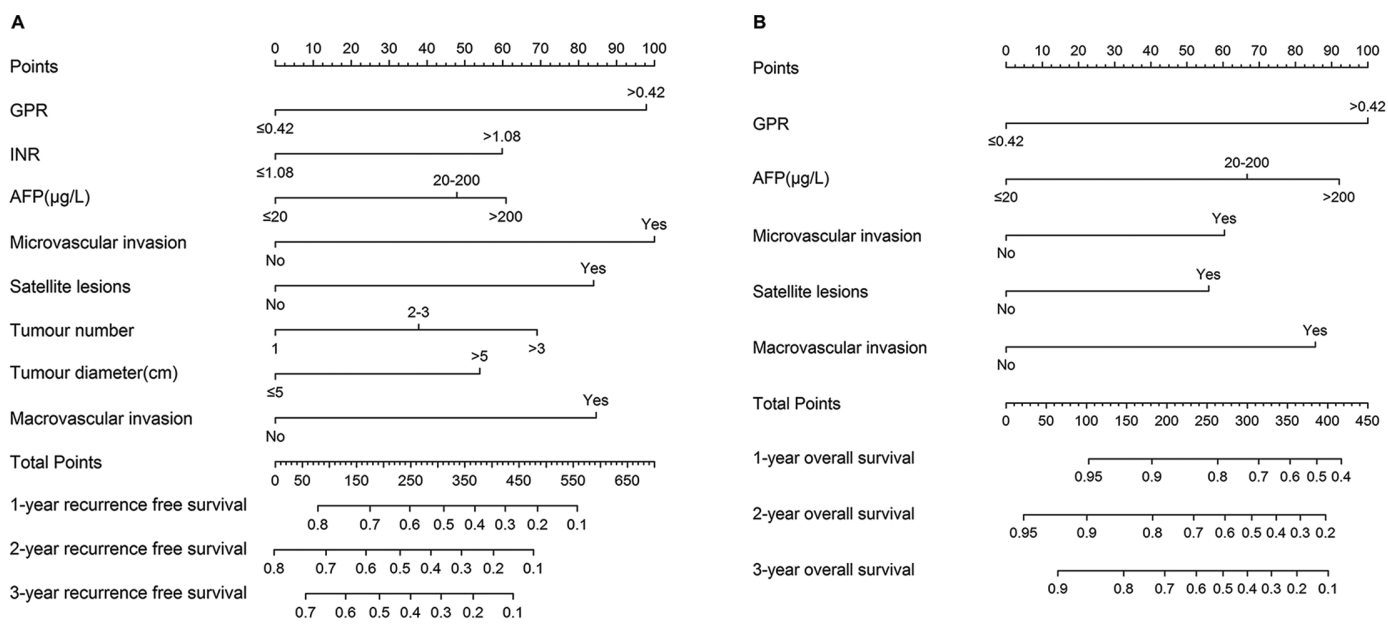

Abstract IDDF2019-ABS-0066 Figure 1 Nomograms for predicting recurrence free survival (A) and overall survival (B)

RFS while GPR, AFP, microvascular invasion, satellite lesions and macrovascular invasion were for OS (figure 1). In the training cohort, the C-index of nomogram was $0.713(95 \%$ confidence interval [CI], 0.690-0.738) for RFS and 0.774 (95\%CI, 0.747-0.801) for OS. These results received both internal and external validation with C-index of 0.707 (95\% CI, $0.662-0.753)$ and $0.710(95 \% \mathrm{CI}, 0.665-0.756)$ for RFS, and 0.747 (95\%CI, 0.686-0.807) and 0.711 (95\%CI, 0.6410.780 ) for OS, respectively. The calibration curves for RFS and OS rates were all well-matched with the standard lines. The nomograms showed superior accuracy to existed staging systems $(P<0.001)$.

Conclusions The novel nomograms based on preoperative inflammation-related markers are of high efficacy in predicting survival of HCC patients after hepatectomy, which will be valuable in guiding postoperative interventions and follow-ups.

\section{IDDF2019-ABS-0070 LIVER RESECTION VERSUS TRANSARTERIAL CHEMOEMBOLIZATION FOR THE TREATMENT OF INTERMEDIATE-STAGE HEPATOCELLULAR CARCINOMA}

'Wanjie Zhu*, ${ }^{2}$ Shuling Chen, ${ }^{3}$ Huilin Jin. ${ }^{1}$ Department of Gastroenterology and Hepatology, The First Affiliated Hospital of Sun Yat-sen University, China; ${ }^{2}$ Division of Interventional Ultrasound, The First Affiliated Hospital of Sun Yat-sen University, China; ${ }^{3}$ Department of Liver Surgery, The First Affiliated Hospital of Sun Yat-sen University, China

10.1136/gutjnl-2019-IDDFabstracts.253

Background The role of transarterial chemoembolization (TACE) as the standard therapy for intermediate-stage hepatocellular carcinoma (HCC) is being challenged by increasing studies supporting liver resection (LR), but evidence of survival benefits of LR is lacking. We aimed to compare the overall survival (OS) of LR with that of TACE for the treatment of intermediate-stage HCC in cirrhotic patients.

Methods A Markov model, comparing LR with TACE over 15 years, was constructed based on data from 31 literatures. Additionally, external validation of the model was performed using a data set $(n=1735$; LR: 701; TACE: 1034) from a tertiary center with propensity score matching method. We conducted one-way and two-way sensitivity analyses, in addition to a Monte Carlo analysis with 10,000 patients allocated for each arm.

Results The mean expected survival periods and survival percentages at 5 years were 77.8 months, $47.1 \%$ in LR group, and 48.6 months, $25.7 \%$ in TACE group, respectively. Sensitivity analyses demonstrated that initial LR was the more favorable treatment. The $95 \%$ CI for the difference in OS was 2.42-2.46 years between the two groups $(P<0.001)$. In the validation set, the 5 -year survival proportions after LR were significantly better than those after TACE before $(40.2 \%$ vs. $25.9 \%, P<0.001)$ and after matching $(43.2 \%$ vs. $30.9 \%$, $P<0.001$ ), which was comparable to the model results.

Conclusions LR may provide a survival benefit over TACE for cirrhotic patients with intermediate-stage HCC.

\section{IDDF2019-ABS-0076 QUALITY OF CARE AND COST ANALYSIS FOR CIRRHOSIS MANAGEMENT IN A LARGE UNIVERSITY-BASED HOSPITAL IN THAILAND}

${ }^{1}$ Kanit Bunnag* ${ }^{*}$ Nattawit Seetalarom, ${ }^{1}$ Amarat Kongsompong, ${ }^{3}$ Sakkarin Chirapongsathorn. ${ }^{1}$ Department of Medicine, Phramongkutklao Hospital, Thailand; ${ }^{2}$ Computer Center, Phramongkutklao Hospital, Thailand; ${ }^{3}$ Division of Gastroenterology and Hepatology, Department of Medicine, Phramongkutklao Hospital, Thailand

\subsection{6/gutjnl-2019-IDDFabstracts.254}

Background As one of the leading causes of death in Thailand, the accurate data about the quality of care, costs and outcome of cirrhotic patients are needed. The aims of this study were to examine the quality of care according to the American Association for the Study of Liver Diseases (AASLD) practice guidelines, costs and outcome in cirrhotic patients in a large university-based hospital.

Methods After IRB approved, cohort study was conducted involving all outpatients with cirrhosis in Phramongkutklao hospital during 2017 through 2018. Medical database and 